
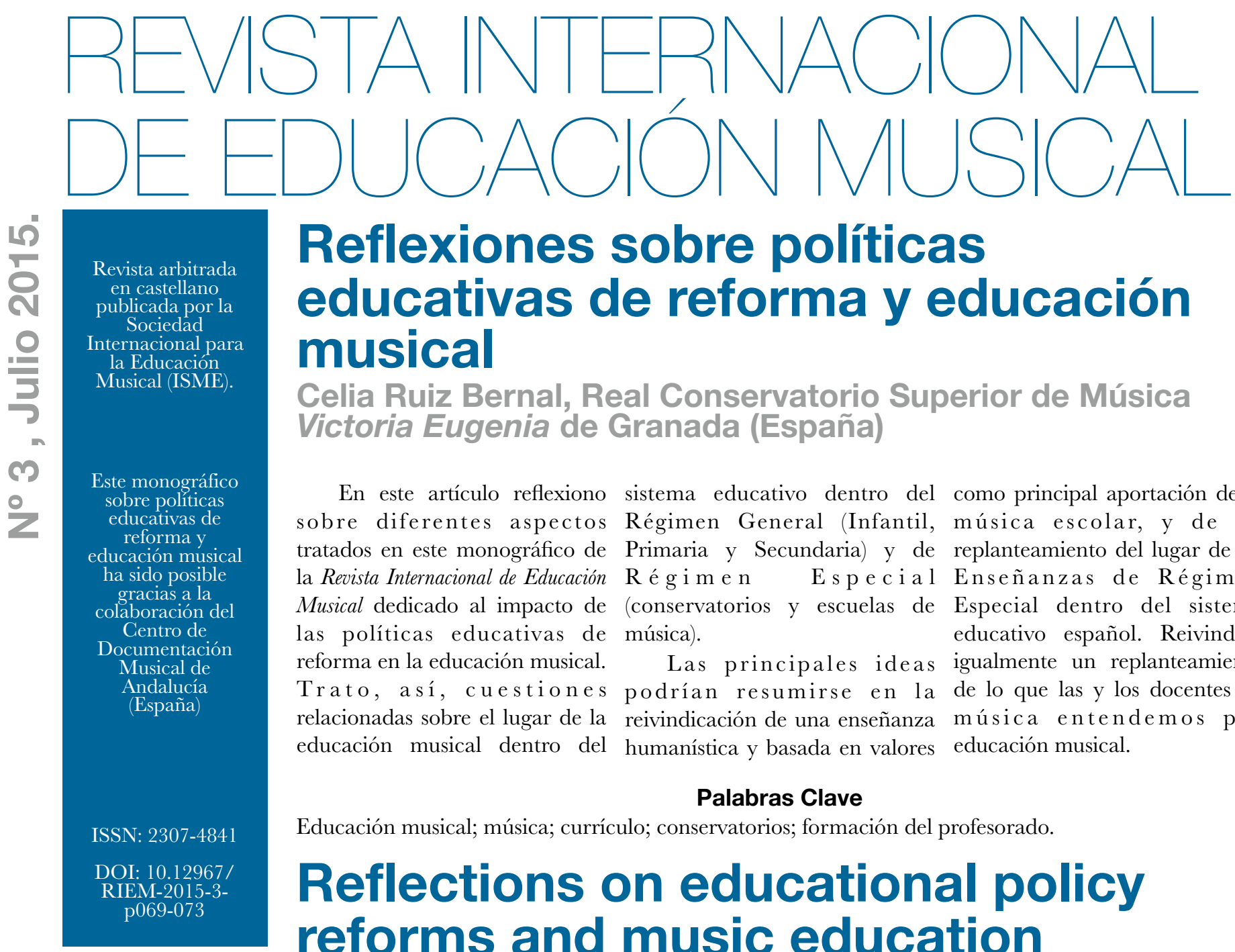

\title{
Reflexiones sobre políticas educativas de reforma y educación musical
}

\section{Celia Ruiz Bernal, Real Conservatorio Superior de Música Victoria Eugenia de Granada (España)}

En este artículo reflexiono sistema educativo dentro del como principal aportación de la sobre diferentes aspectos Régimen General (Infantil, música escolar, y de un tratados en este monográfico de Primaria y Secundaria) y de replanteamiento del lugar de las la Revista Internacional de Educación Rég ime n E s p e c i a l Enseñanzas de Régimen Musical dedicado al impacto de (conservatorios y escuelas de Especial dentro del sistema las políticas educativas de música). educativo español. Reivindico reforma en la educación musical. Las principales ideas igualmente un replanteamiento Trato, así, cuestiones podrían resumirse en la de lo que las y los docentes de relacionadas sobre el lugar de la reivindicación de una enseñanza música entendemos por educación musical dentro del humanística y basada en valores educación musical.

\section{Palabras Clave}

Educación musical; música; currículo; conservatorios; formación del profesorado.

\section{Reflections on educational policy reforms and music education Celia Ruiz Bernal, Victoria Eugenia Granada Higher Conservatory (Spain)}

Abstract

In this paper I reflect on Secondary Education) and the contribution of the school music. some topics discussed in this specialised music education I also claim for a reconsideration monograph about educational system (conservatoires and of the place the higher music policy reforms and their impact schools of music). education system takes place in on music education. Thus, I Major ideas provided could the Spanish educational system. cope with issues related to the be summarised in the Finally, I suggest a redefinition of place music education has as vindication of a value-based music teachers understand as part of the general curriculum humanistic education as major music education.

(kindergarten, Primary and

\section{Keywords}

Music education; music; curriculum; conservatoires; teacher education.

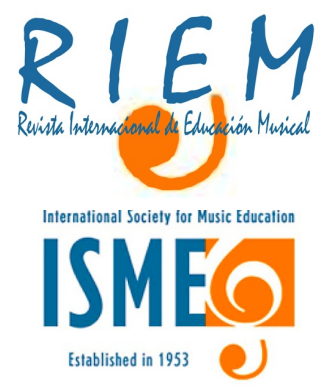


 \\ Reflexiones sobre políticas educativas de reforma y educación musical}

por Celia Ruiz Bernal, Real Conservatorio Superior de Música Victoria Eugenia de Granada (España).

Siempre es buen momento para la reflexión, máxime cuando la humanidad experimenta cambios tan vertiginosos y dramáticos como los que vivimos, y aun sabiendo que son ciclos de depresión y recuperación, debemos poner todos los medios y todo nuestro empeño en conseguir un mundo mejor a todos los niveles.

La educación musical, aunque no suficientemente reconocida y apoyada en nuestro país, tiene sin embargo mucho que aportar a la educación integral y tenemos la obligación de fomentar foros de debate donde tomar conciencia del beneficio que la música aporta al ser humano y a la convivencia, y la importancia de nuestro papel como educadores, sobre todo para que las nuevas generaciones de educadores que se han formado en un contexto que ha cambiado radicalmente y se ven obligados a cambiar los esquemas y patrones educativos a toda velocidad y muchas veces sin la suficiente ayuda experta.

Diariamente leemos sobre la constante preocupación del músico, maestro, investigador, creador e intérprete, en todos los niveles educativos y a todos los niveles sociales y personales por llevar los beneficios de la música al ser humano y a la sociedad, pero las prioridades de la clase política son muy ambiciosas económicamente hablando y muy pobres humanamente aplicada.

A pesar de demostrar constantemente que gracias a un proyecto donde el potencial humano es sobradamente competente y los beneficios están constatados por investigadores e instituciones de prestigio con importantes asignaciones económicas, sigue sorprendiendo que quien dispone sobre política educativa y política social no tiene en cuenta que la aplicación de unos estudios con magníficos resultados aportarían un gran beneficio a la educación de niños y niñas de cualquier país, contribuye a la inclusión social y produce bienestar a lo largo de la vida

Millones de euros son destinados a proyectos para que escolares de todas las edades aprendan música fuera del sistema educativo. Si está demostrado que el beneficio va directamente relacionado con el desarrollo y el rendimiento escolar y social, ¿no sería más lógico tener la práctica musical integrada en la enseñanza obligatoria como una disciplina más? Es claro que no es suficiente compartirla en el área de educación artística, sino que necesita de su propio espacio y tiempo como un lenguaje más, que permite la comunicación y la expresión.

Proyectos educativos y musicales, resultados, formación, integración, investigación, son piezas de un puzzle que los países intentan resolver sin perspectiva. Vemos por ejemplo cómo en el Reino Unido existen grandes proyectos musicales subvencionados con dinero público para que los niños aprendan a cantar, sin embargo la práctica musical como disciplina queda a criterio de la propia escuela.

Otro reto que compartimos los educadores de música de todos los países es la situación de aislamiento en la que trabajamos, a veces por las circunstancias o la propia organización del centro educativo, y otras por nosotros mismos, que sumergidos en una inercia del trabajo solitario, tiene como resultado la dificultad para integrarnos en un colectivo de docentes con el que tenemos que compartir nuestro tiempo, el alumnado, las inquietudes, los proyectos, las ilusiones, que dicho sea de paso, sería compartir experiencias, dificultades y éxitos.

Esta situación de aislamiento no puede hacernos perder de vista que por encima de las situaciones, hemos de seguir convencidos y demostrando que estamos seguros de los valores y beneficios de la música para, trabajando en equipo, poder convencer a los políticos que nos gobiernan $y$ conseguir los cambios que mejoren el sistema.

Llevamos quince años en el siglo XXI y todavía la música es considerada un juego, que no es poco, pero si está demostrado que aporta beneficios al ser humano ¿por qué limitar su función? No se concibe el mundo sin música, es una capacidad espontánea que el ser humano posee desde que nace igual que la capacidad de hablar. ¿Por qué no está en la escuela al mismo nivel que otras disciplinas?

¿Dónde radica el éxito de otros sistemas educativos como vemos en los informes que se publican periódicamente? Nos estamos empeñando en educar en el siglo XXI con fórmulas y herramientas del siglo XX, o lo que es peor, del siglo XIX. De todos es sabido, pero, ¿por qué no empezamos desde dentro a propiciar el cambio? Ahora que nos animan a investigar y a innovar, ¿por qué ese miedo del docente a salir fuera de la «tradición»? Tenemos en nuestras manos la capacidad de formar con diferentes patrones, por ejemplo a través de las TIC, y sin embargo no siempre le sacamos el partido necesario. Bien es cierto que la administración ha puesto su granito de arena, pero hagamos examen de conciencia, ¿hemos puesto nosotros, el profesorado todo de nuestra parte? Quizás nos da miedo que el alumnado tenga más conocimientos TIC que nosotros mismos. ¿Y por qué no aprovechar y construir desde ese conocimiento previo?

Somos conscientes de que la política educativa está construida de dentro a fuera de la calle y no estamos educando ni enseñando para la sociedad de hoy, ni para la sociedad del mañana.

El mundo global se aleja cada vez más de la Cultura y los valores propios del ser humano. Mientras unos pocos hacen grandes esfuerzos por recuperar y mantener cultura y tradiciones, quienes poseen las herramientas de difusión dedican todo su esfuerzo a embrutecer y desensibilizar a una humanidad a través de situaciones irreales, nada interesantes, ni formativas, ni siquiera entretenidas, alejadas de la ética y los valores básicos del hombre.

Tras muchas horas de debate y reflexiones hemos caído en la cuenta de que nos inculcan lo que se vende, hemos sido demasiado lentos y apáticos, hasta ahí somos conscientes. La crisis que sufrimos en las aulas es la crisis de la calle, la crisis de la clase política, una brutal ausencia de valores éticos, todo vale y el dinero lo compra todo. Es muy difícil cambiar a toda una sociedad, pero somos los educadores, los profesionales por excelencia de establecer «valores» que hagan del ser humano un ser «superior». 
Por otra parte, países como España luchan por mantener un sistema de formación del profesorado mientras ve cómo la Administración está dejando desaparecer la docencia de la música en las aulas de la enseñanza obligatoria. ¿Alguien se ha parado a pensar que a millones de niños se les está privando del derecho a un mejor desarrollo como persona? Otra fatuidad de la clase política, que ha pensado que regalando ordenadores va a conseguir mejores ciudadanos. Primero hay que inculcar valores para después saber apreciar lo que tenemos. La música es solidaridad, es cuidar y acariciar un instrumento que tiene vida propia, la música es compartir, es saber esperar, saber callar, saber emocionar... la música es un valor. ¿Qué habría pasado si el Ministerio de Educación hubieses regalado instrumentos musicales?

Son muchos los esfuerzos, en todas las instituciones donde la música tiene la categoría y el respeto que se merece, y desde donde constantemente se levanta la voz, aun siendo un colectivo pequeño. ¿Podemos imaginarnos una escuela infantil sin música? Estamos privando a los niños y las niñas de la forma de expresión de sentimientos más natural que posee.

Pero los educadores tenemos que hacer más, no podemos limitarnos a leer artículos donde se pide a gritos el cambio sin hacer nada, participar en intercambios de formación donde todo queda en una declaración de intenciones, de reformar guías docentes para continuar con el discurso rancio que ya no responde a las necesidades educativas del alumnado.

Es evidente el declive que está sufriendo la música en las enseñanzas obligatorias con la aplicación de las nuevas políticas educativas. Y hay que seguir haciendo ver a la sociedad que la música es calidad de vida, que gracias a ella no sólo el desarrollo del niño es mejor, sino que es potencial de expresión, de juego, de comunicación, de aprendizaje, de relajación, de sanación. Hay que mantener la necesidad de la música en la sociedad, para que cada vez seamos más los beneficiados, pero eso requiere el esfuerzo de todos nosotros.

Somos el profesorado en general el que debe ser consciente y creer en la música como lenguaje de comunicación y expresión y de los beneficios que aporta.

Investigación e innovación son dos términos que nos deben acompañar siempre. La sociedad cambia, las preferencias, gustos, necesidades. Somos artistas y en mayor o menor medida nuestra capacidad de crear y transmitirlo a niños y niñas tiene que hacer honor a ello.

Es cierto que existen malas prácticas en las escuelas, hay que impulsar la puesta en marcha de nuevas ideas. Afortunadamente existe la autonomía suficiente como para crear nuestro propio proyecto de cómo enseñar lo que queremos y debemos enseñar. La investigación es trabajo del docente, somos nosotros los que tenemos que realizar los cambios en el aula, sobran teorías y faltan ideas prácticas para mejorar la actividad docente y eso es responsabilidad nuestra, sin miedo y creyendo en nuestras capacidades.

Los educadores tenemos que reflexionar seria y profundamente porque es necesario encontrar nuevos esquemas que desafien la teoría y la praxis cotidiana y habitual. Poner en práctica nuevas ideas, nuevas acciones, nuevas verdades que resulten más útiles en tiempos de cambios como el que vive la humanidad. Esta reflexión basada en el examen de la práctica diaria, es la base para encontrar nuevos caminos de acción.
El mundo está en permanente cambio, en permanente transformación y en una profunda crisis de valores. Aprovechemos la creatividad que emana de ese cambio para potenciar los valores que la música aporta al ser humano, promoviendo un cambio social positivo.

Debemos reflexionar también sobre el papel que desempeñan hoy por hoy las enseñanzas superiores de la música y las artes escénicas, dónde están ubicadas y cuál es su papel en la sociedad.

Aunque históricamente no ha sido así, en la Europa y América del siglo XXI, las enseñanzas superiores de música se imparten tanto en universidades como en conservatorios, escuelas superiores y academias, manteniendo el estatus de enseñanzas superiores, pero no en todos los países se ha tenido el cuidado de dejar claramente determinado ese espacio de educación superior que ocupan y en el que conviven con el resto de enseñanzas superiores.

En nuestro país, en este tema de nuevo a la cola de Europa, aunque las Enseñanzas Artísticas Superiores están ubicadas por Real Decreto en el nivel de grado universitario, se siguen denominando Título Superior, lo que hace necesaria la explicación de «equivalente a». Y gracias a una legislación eternamente inacabada, la enseñanza musical superior sólo alcanza la categoría académica de Grado y Máster, dejando el Doctorado para realizar en convenio con las Universidades donde paradójicamente la Música va desapareciendo del catálogo de las titulaciones.

El problema de no estar en el mismo espacio de Educación Superior alimenta situaciones de incómoda convivencia como es el caso de la duplicidad de titulaciones que aparecen en conservatorios superiores y universidades, pudiendo darse la incongruencia de no poder colaborar por pertenecer a administraciones diferentes. De nuevo vemos como un tema mal resuelto políticamente perjudica a colectivos que deberían estar trabajando juntos por un mismo objetivo.

Aunque estamos hablando de un colectivo relativamente pequeño a nivel nacional, tenemos que despertar la conciencia colectiva, ya que todos trabajamos en pro de la educación musical en las enseñanzas obligatorias y de régimen especial, como maestros de música en las enseñanzas obligatorias, como músicos profesionales en los conservatorios y escuelas de música, como formadores de futuro profesorado en las universidades.

No podemos perder de vista qué queremos conseguir y cómo de grande es nuestro compromiso ético, desde las instituciones, desde la sociedad, desde los centros de enseñanzas artísticas y universidades y desde la individualidad de cada uno de nosotros.

La ley de Mecenazgo, una oportuna y necesaria Ley, debe suponer el compromiso de colaboración de la sociedad para con estas enseñanzas, su desarrollo y proyección. Y a su vez las Enseñanzas Artísticas Superiores tienen el compromiso con la sociedad en la preparación de los profesionales competentes para desarrollar estas enseñanzas en el siglo XXI, con sus nuevos perfiles, exigencias y necesidades.

Desde nuestro puesto de trabajo y mientras los cambios sociales dan paso a etapas de consolidación, nuestra aportación personal y nuestro grado de compromiso personal siempre será el cimiento que mantendrá el éxito en nuestro 
เด็ trabajo como educadores en la música, desde la música, para

ำ la música y por la música.

क

ㄴ.

نं

$\frac{\mathbb{U}}{0}$

D

$\stackrel{2}{\gtrless}$

$\frac{Z}{0}$

ర

$\circlearrowleft$

뭄

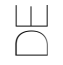

3
$\frac{1}{0}$
$\frac{1}{4}$
$\frac{1}{3}$
$\frac{1}{4}$
$\frac{0}{11}$
0 


\section{Sobre la Autora}

\section{Celia Ruiz Bernal}

Celia Ruiz Bernal. Titulada Superior en Piano y Máster en Musicoterapia. Fue docente en los Conservatorios Superiores de Málaga y Sevilla. Es Catedrática de Solfeo y Teoría de la Música, y autora de varias publicaciones didácticas. Actualmente es Directora del Conservatorio Superior de Música de Granada y miembro del Consejo Superior de Enseñanzas Artísticas del Ministerio de Educación, Cultura y Deporte y del Consejo Andaluz de Enseñanzas Artísticas Superiores.

\section{Celia Ruiz Bernal}

Conservatorio Superior de Música Victoria Eugenia de Granada

San Jerónimo, 38

18001 Granada. España.

mcruizbernal@gmail.com

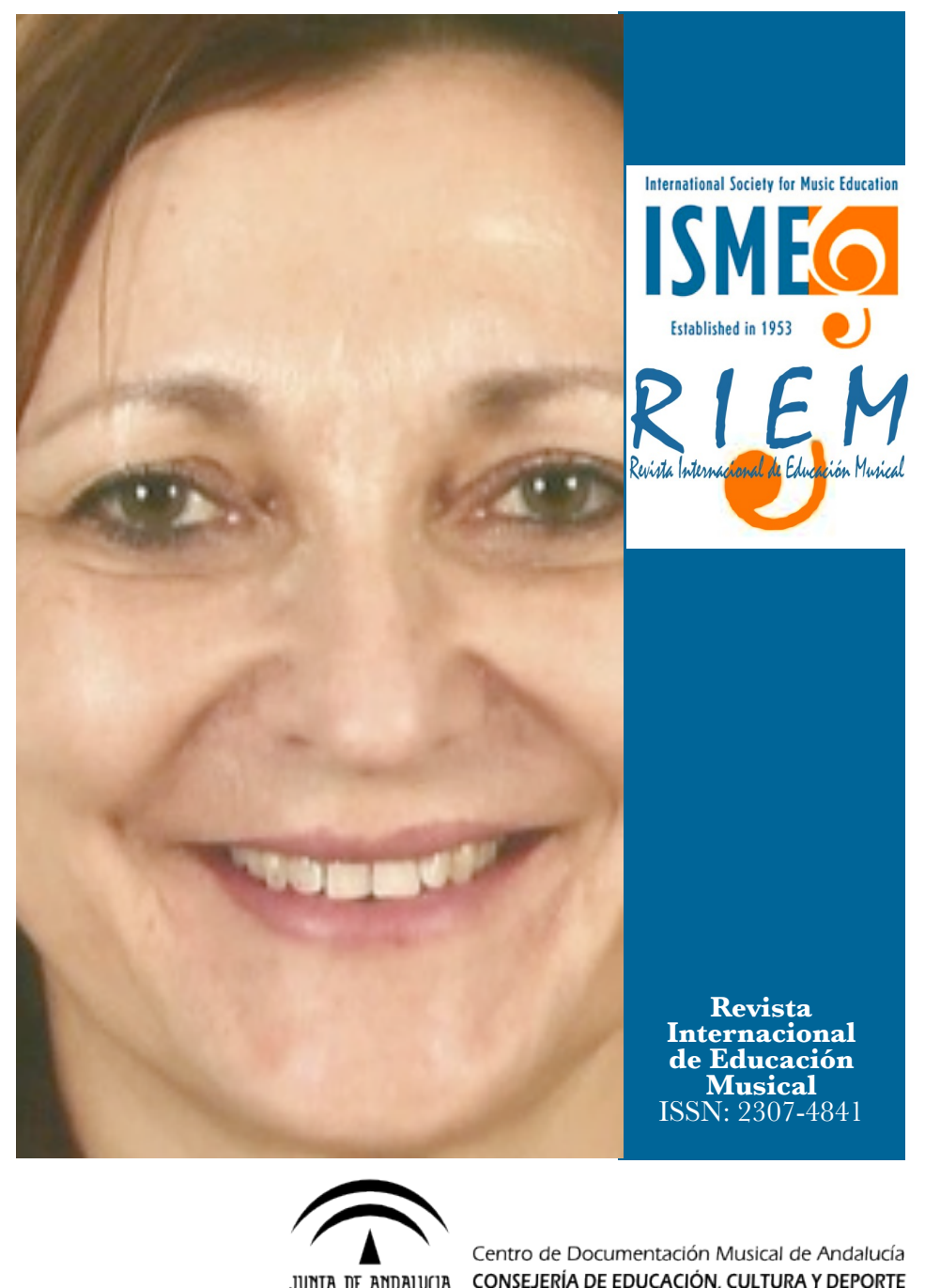

JUNTA DE AMDALUCIA CONSEJERIA DE EDUCACIÓN, CULTURA Y DEPORTE

\section{EQUIPO EDITORIAL}

Editor:

José Luis Aróstegui Plaza, Universidad de Granada (España)

\section{Editora Adjunta:}

Rosa María Serrano Pastor, Universidad de Zaragoza (España)

\section{Consejo Editorial}

Carlos Abril, Universidad de Miami (Estados Unidos)

María del Carmen Aguilar, Instituto Coral de Buenos Aires (Argentina)

Miquel Alsina Tarrés, Universidad de Gerona (España)

Carmen Carrillo Aguilera. Universidad Internacional de Cataluña (España)

Sergio Luiz Figueiredo, Universidad del Estado de Santa Catarina (Brasil)

Patricia Adelaida González, Universidad Autónoma de Chihuahua (México)

Claudia Gluschankof, Instituto Levinsky (Israel)

Josep Gustems Carnicer, Universidad de Barcelona (España)

María Cecilia Jorquera Jaramillo, Universidad de Sevilla (España)

Gotzon Ibarretxe Txakartegi, Universidad del País Vasco (España)

Ana Laucirica Larrinaga, Universidad Pública de Navarra (España)

Ana Lucia Louro, Universidad Federal de Santa María (Brasil)
Isabel Cecilia Martínez, Universidad Nacional de La Plata (Argentina)

Teresa Mateiro, Universidad del Estado de Santa Catarina (Brasil)

María Teresa Moreno, Universidad Laval (Canadá)

Graça Boal Palheiros, Instituto de Educación de Oporto (Portugal)

Jèssica Pérez Moreno, Universidad Autónoma de Barcelona (España) y Universidad de Londres (Reino Unido)

Gabriel Enrique Rusinek Milner, Universidad Complutense de Madrid (España)

Patrick K. Schmidt, Universidad de Ontario Occidental (Canadá)

Favio Shifres, Universidad Nacional de La Plata (Argentina)

Maria dels Àngels Subirats Bayego, Universidad de Barcelona (España)

António Ângelo Ferreira Vasconcelos, Instituto de Educación de Setúbal (Portugal)

Maria Helena Vieira, Universidad del Miño (Portugal)

Gloria Patricia Zapata Restrepo, Universidad de Antioquía (Colombia) 